will prove of value in dealing with a considerable number of complications involving fecal fistula. It was my original intention to have left the provisional pursestring suture undisturbed, thus placing the closed orifice of the perforation in the floor of the colostomy wound, with the hope that the patency of the colon lumen would have been re-established and rapid repair have been accomplished. During the manipulation, however, the provisional suture was torn out and an artificial anus was established at once.

Incidentally, I wish to express my high appreciation, of the work done by F. Gregory Connell, bearing upon the matter of intestinal suture as described by him. (The Journal 1. M. A., Oct. 12, 1901.) I have thus far employed th. Connell method in two cases with the utmost satisfaction. Its technique when once fully understood is simple and its various steps can be completed with a fair degree of rapidity. So far as safety is concerned. I believe it is capable of demonstration that the placing of the knot within the lumen of the bowel is a most important consideration.

406 Sutter Street.

\section{A CASE IIILUSTRATING PLASTIC SURGERY OF THE EYELIDS.}

CASSIUS D. WESCOTT, M.D.

Assistant Professor of Ophthalmology at Rush Medical College ; Assistant Ophthalmologist to the Presbyterizn Hospital, etc.

$$
\text { cHICAgo. }
$$

During my service at the Presbyterian Hospital in the summer of 1900, Miss Sarah McB., aged 38, presented

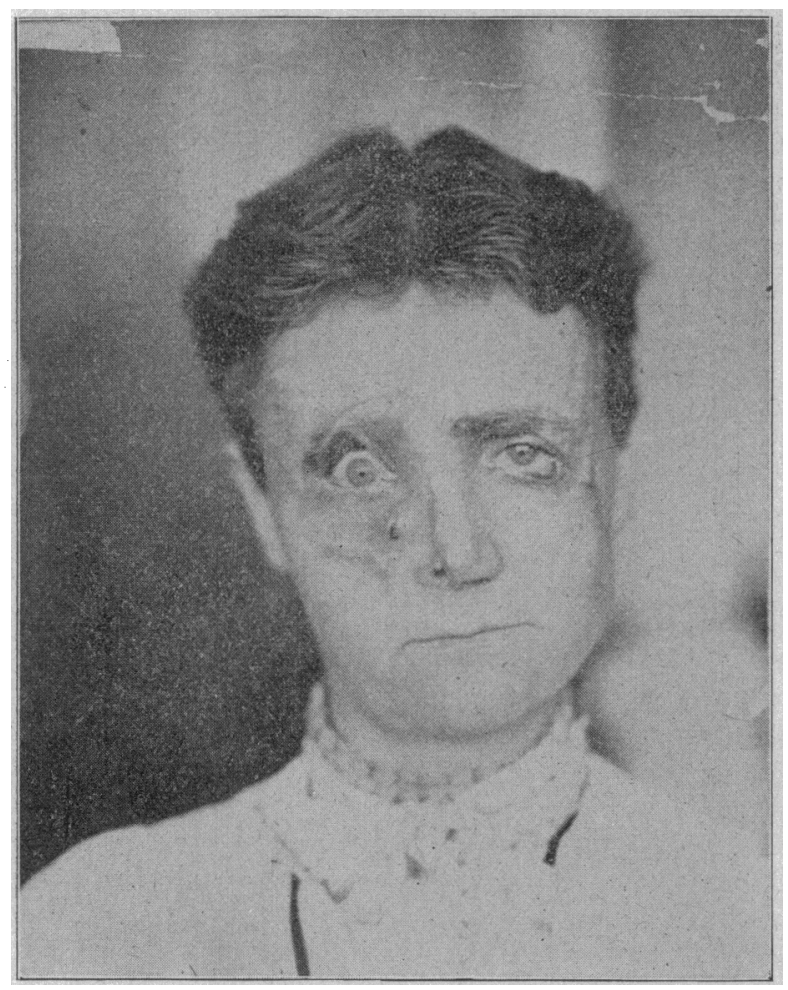

Fig. 1.-August 6. After first operaticn on cheek.

herself for the correction of deformities of the eyelids resulting from burns, which she had received six months before by falling into a grate fire in a fainting fit. As shown in photograph No. 1 , the soft parts had suffered severely on both sides of her face, a portion of the right wing of the nose had been lost, and there was complete ectropion of the upper lia of the right eye and almost complete eversion of the lower lid of the left eye. The brow just over the nose was so deeply burned that a portion of the external surface of the frontal bone had necrosed and come away during the dressing of the burns. The new skin which had formed in this region and over the right eye was extremely thin and poorly nourished, and the ciliary border of the lid was tightly

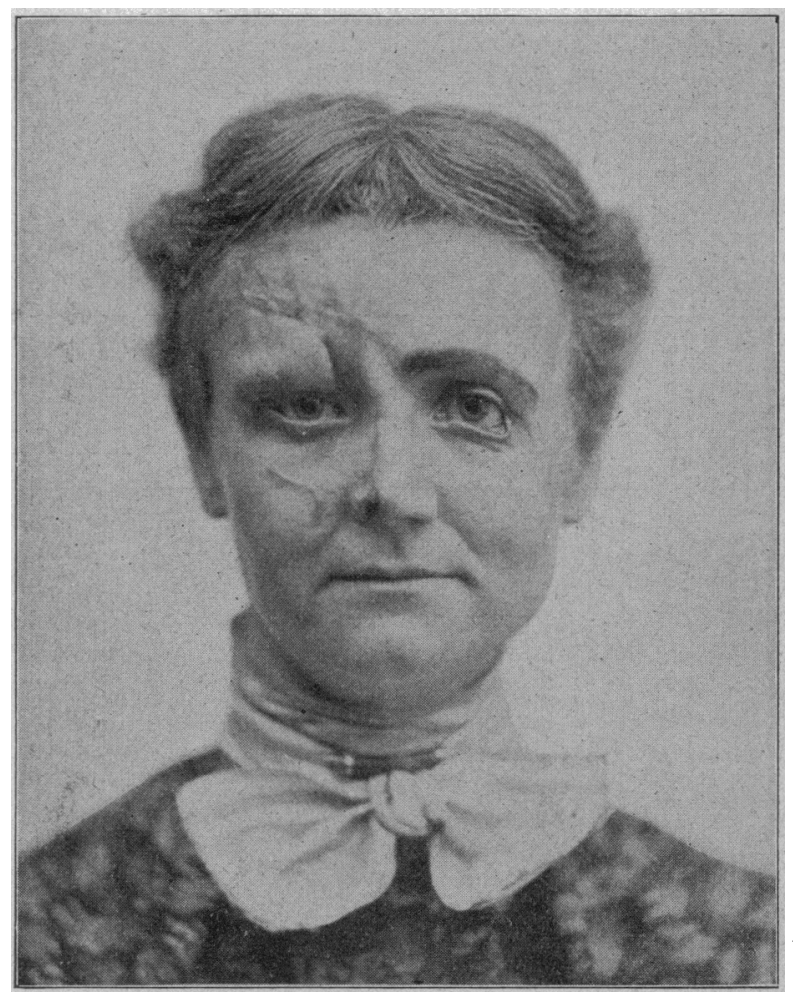

Fig. 2.-September. One month after operation for restoration of upper lid of the right eye.

drawn up and firmly adherent to the periosteum. In the left cheek was a very thick keloid scar, which sent an arm to the lower lid of the left eve, dragging it down and out and away from the globe. The cornea of the right eye was constantly exposed to dust and the danger of trauma and only an excessive flow of tears, which kept the epithelium moist and washed away the dust, saved it from inflammation, ulceration and the usual sequelæ.

As there was a broken-down and suppurating area over the right brow, it was not deemed advisable to begin work there, and the first operation consisted in the removal of the large scar of the left cheek and the restoration of the lower lid of the left eye to its normal position, by a free dissection and crowding up of the entire cheek. This was done July 6 and the immediate result was quite perfect; but, as so often occurs in plastic surgery in all parts of the body, a portion of our flap sloughed near the outer end of the lid and the final result was an ectropion almost as complete as there was in the beginning. The condition of the patient's heart and kidneys did not permit of another anesthesia until August 15, when I made a free dissection of the upper lid of the right eye, stitching it to the lower lid and bringing down a large flap from the forehead, with pedicle on the temple, to cover the extensive surface exposed. After this flap from the brow was carefully stitched in place, the area from which it was taken was filled in with Thiersch grafts, as shown in photograph No. 2, which was taken one month later. Healing after this operation was very satisfactory, and on October 6 
the left lower lid was restored to its normal position by making a large $V$-shaped incision below and to the outer side and converting this $\mathrm{V}$ into a $\mathrm{Y}$ by freely dissecting and crowding the flap up, thus forcing the free edge of the lid into its proper position. Healing was slow, but the ultimate result has been good. Both eyes are closed during sleep and photograph No. 3 shows the patient's appearance one year after the operation on the right eye.

When all tenderness had disappeared from the scars, the patient was referred to $\mathrm{Mr}$. Hugo Ad. Oldenborg for massage, and there is good reason to believe that his careful and systematic treatment was of great assistance in obtaining the excellent final result.

I am also indebted to Dr. A. I. Bouffleur for his valuable assistance in my operations, and for undertak-

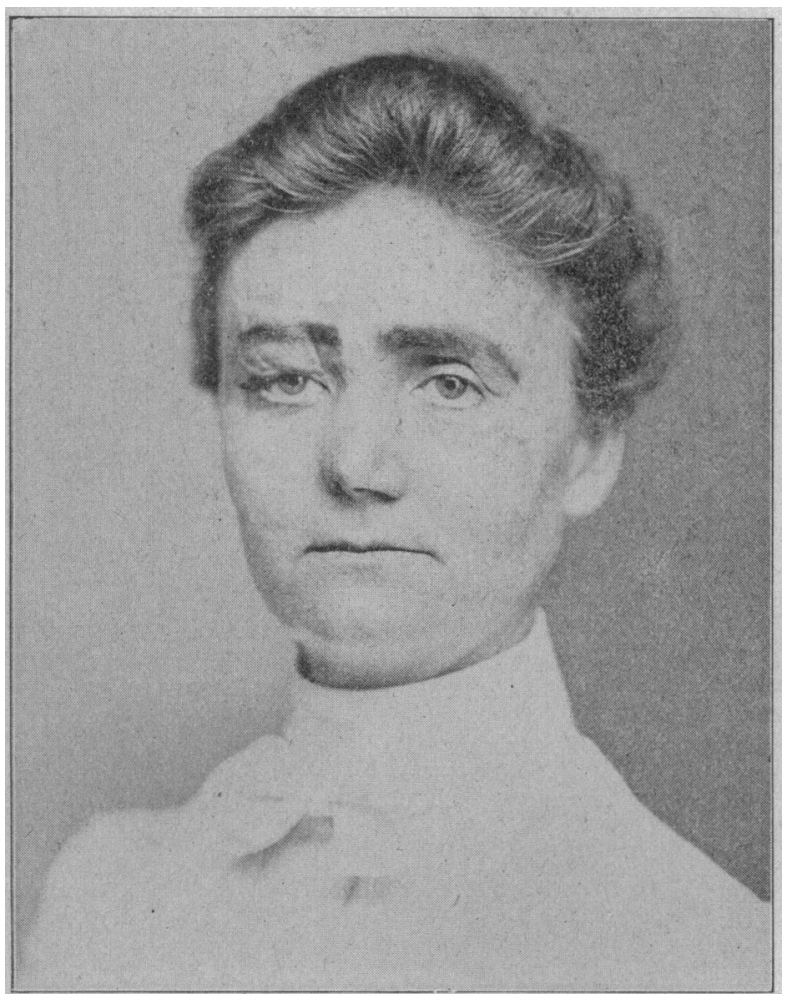

Fig. 3.-September, 1901. One year after last operation.

ing the repair of the nose; the appearance of which he improved very much.

31 Washington Street.

\section{THE X-RAY IN DETERMINING THE LIMITS OF THE FRONTAL SINUS. JOHN HAROLD PHILIP, M.D. \\ SAN FRANCISCO.}

In the June number of the Laryngoscope I note the following from the pen of Dr. Jonathan Wright: "I have lately seen the frontal bone perforated and the dura mater wounded with disastrous results in a case in which the frontal sinus was lacking on that side."

Dr. Howard A. Lothrop, of Harvard University, after carefully examining 250 frontal sinuses, from dissecting room material, concluded that there was no external landmark defining the superior limit of the sinus; that the external angular process of the frontal bone was not often the limit of the sinus laterally; that in the majority of cases the septum (between the sinuses) deviated to one side or the other within a range of five millimeters or even more, notwithstanding its frequent median position inferiorly; that the plane of the septum was roughly antero-posterior, passing between the anterior and posterior surfaces, but occasionally it might so deviate that one sinus would lie partly overlapping the other, even to. an extent of 2 centimeters; that there were no absolutely certain guides by which the degree of development of the frontal sinus in the adult could be determined before attempting to expose it.

Previous to entering a frontal sinus some months since I secured three frontal bones and attempted to explore with my drill that sinus corresponding to the one on which I was to operate. I was amazed to find that No. 1 had almost no sinus on the left side; that the sinus on the left side of No. 2 extended not more than 3 milli-

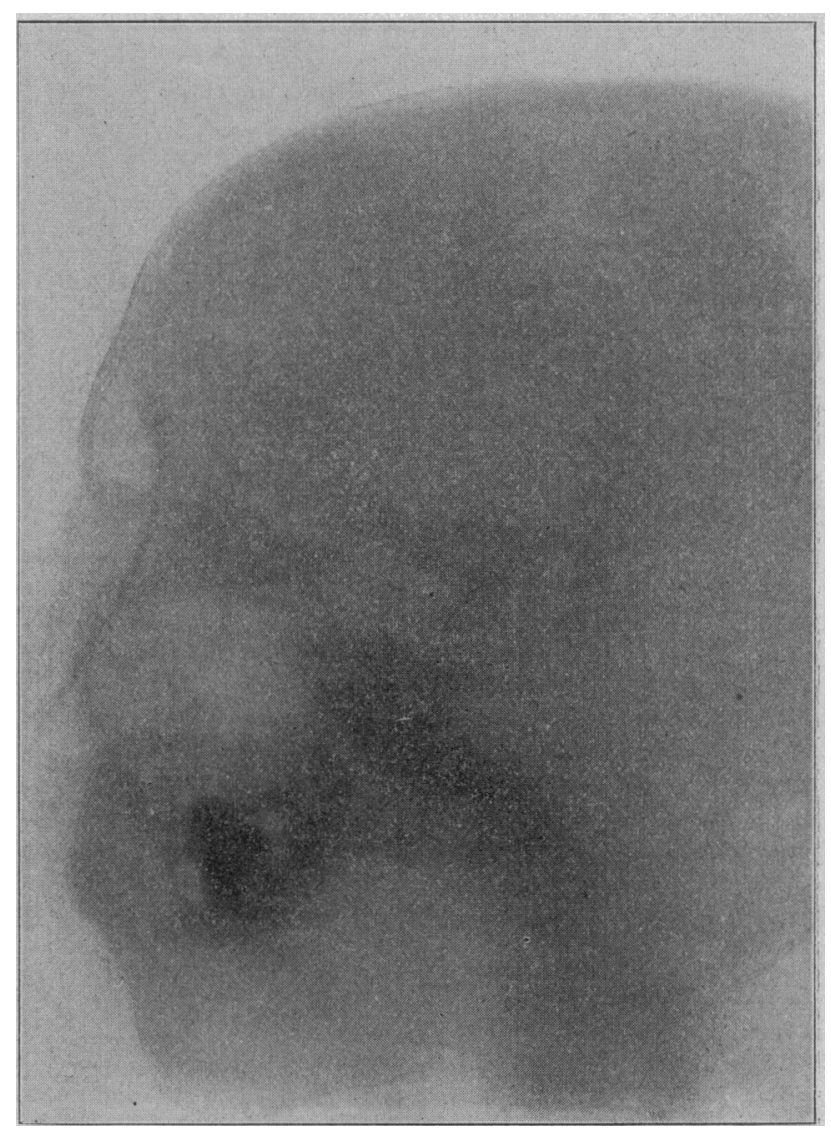

meters beyond the median line and that there was no septum; there was no communication with the left nasal cavity. No. 3 seemed normal. One can not well overestimate the surgical importance of such anatomical anomalies, whose frequency is unquestioned.

As an aid to determining. previous to operation, the limitations of the frontal sinuses I suggest the $x$-ray.

The radiograph illustrating this article was taken for me by Mr. Cox of the San Francisco Polyclinic. Length of exposure was eight minutes; distance of tube from plate, 20 inches; plate over-developed and the print was sun-printed and overtoned. My patient's head lay obliquely on the plate and the affected sinus Iay undermost. This explains the position of the camula, its tip extending apparently beyond the posterior wall of the sinus. For cosmetic reasons I entered the sinus from within the nasal cavity. (My patient was a young woman of 24.) Wishing to be certain that my canula was not in an anterior ethmoidal celI. I had a radio- 